\title{
Putting Restraint on Chemical Restraint: Exploring the Complexity of Acute Inpatient Mental Health Nurses' Experiences of Chemical Restraint Interventions
}

\author{
Michelle C. Danda, RN, PhD (c) \\ University of Alberta, School of Nursing
}

Cite as: Danda, M. (2020). Putting Restraint on Chemical Restraint: Exploring the Complexity of Acute Inpatient Mental Health Nurses' Experiences of Chemical Restraint Interventions. Witness: The Canadian Journal of Critical Nursing Discourse, Vol 2(2), pp 29-53. https://doi.org/ 10.25071/2291-5796.75

\begin{abstract}
While there is a growing body of research available on general restraint intervention in acute adult psychiatric settings, relatively little is known about nurses' experiences of administering chemical restraint. The research question explored in this study was: What are mental health nurses' experiences of using chemical restraint interventions in times of behavioural emergency on adult inpatient acute mental health units? Understanding of direct care nurses' first-hand experiences of the use of chemical restraint interventions was sought in this Canadian study. Eight adult acute inpatient mental health nurses were interviewed using a interpretive phenomenological methodology. Two major themes that emerged from data analysis are explored to illuminate the existing tension between therapeutic, person-centred care and coercive control to maintain safety: taking control to maintain safety and working within constraints. Study findings uncovered integral ways that nurses made meaning of their experiences administering chemical restraint. Critical analysis explores the complex clinical and ethical decision-making aspects involved in mental health nurses' use of this coercive intervention. Implications for practice, education, and policy are discussed. Research findings indicated a need for further focus on medication best practice, policy development, and nurse education inclusive of multiple perspectives, most importantly, the patient's. These exploratory research findings can be used to both inform and challenge dominant inpatient mental health practice to guide nurses, health care leaders, and policy makers by increased understanding of the complex ethical decision making required for use of chemical restraint interventions.
\end{abstract}

Key Words: Chemical restraint, coercive medication, restraint practice, phenomenology, mental health nursing 


\section{Background}

There are three types of restraint typically used in acute mental health inpatient settings: physical, environmental, and chemical (Emmanuel et al., 2013; Keser Özcan et al., 2015; National Consumer and Carer Forum, 2009). Restraint use has been a common nursing practice in acute inpatient mental health care settings (Chieze et al., 2019; Garriga et al., 2016; Landeweer et al., 2010). For decades, the common practice on acute inpatient mental health units was guided by guidelines and educational material for mental health staff that highlighted the coercive use of psychotropic Pro Re Nata (PRN) medication to subdue patients who pose a safety risk, most notably violence risk (Emmanuel et al., 2013; National Consumer and Carer Forum, 2009; Registered Nurses' Association of Ontario, 2012), despite little published formal research on the use of chemical restraint interventions until recent years $(\mathrm{Hu}$ et al., 2019; Muir-Cochrane, 2020; Muir-Cochrane et al., 2019; Muir-Cochrane, Oster, \& Grimmer, 2020; Muir-Cochrane, Oster, Gerace, et al., 2020). Direct care nurses are most often the health care providers who initiate and administer the coercive interventions of restraints on mental health inpatient units (Bigwood \& Crowe, 2008; Cunha et al., 2016). There is a growing body of literature identifying the need for more research on coercive practices in mental healthcare settings (Hui et al., 2013; Perkins et al., 2012; Soininen et al., 2013). Research is widely available on general restraint intervention (Cannon et al., 2001; Gelkopf et al., 2009; Ling et al., 2015), in some of which research the authors explored patient (Georgieva et al., 2012) and nurse (McCain \& Kornegay, 2005) experiences, yet relatively little is known about nurses' experiences of use of chemical restraint specifically.

The purpose of this paper is to present the major findings of a master's research project aimed at gaining insight into the experiences of mental health nurses' use of chemical restraint in managing behavioural emergencies in the adult inpatient mental health care setting. Two major themes that emerged in data analysis: 1) taking control to maintain safety, and 2) working within constraints, will be used to illustrate the complexity of mental health nurses' attempts to reconcile the discordance between application of this coercive intervention in the name of safety, while also attempting to embody a therapeutic and patient-centred approach within a workplace rife with limitations. A brief literature review is provided to contextualize the central research question, followed by explanation of the research design. Analysis presents the thick rich data and emergent subthemes. Discussion is focused on unpacking the complexity of ethical issues nurses faced, contextualizing it within the greater chemical restraint research landscape. Lastly, recommendations for policy and education change are provided, and strengths and limitations of this research are explored.

\section{What is Chemical Restraint? Exploring the Research Question}

The phenomenon of interest was nurses' experience of the use of chemical restraint interventions for managing behavioural emergencies with adult patients on acute inpatient mental health units. Behavioural emergencies are broadly defined as, "describing symptoms of acute behavioral distress experienced by patients, including those on inpatient medical or surgical units. Behavioral emergencies comprise 3 distinct subtypes: clinical psychiatric emergencies, coping/stress reactions, and conflicts due to iatrogenic insults" (Parker et al., 2020, p. 957). The research question was: What are mental health nurses' experiences of using chemical restraint interventions in times of behavioural emergency on adult inpatient acute mental health units? The research goal was to learn about nurses' perspectives to increase understanding of the ways that nurses made meaning of medication practices commonly considered chemical restraint interventions vis- $a$-vis the clinical and ethical decision-making process.

Interpretive phenomenology was determined as best suited to explore the phenomenon of chemical restraint experiences because this research methodology gave voice to the nurses, who regularly used the practice, and provided insight into the nuances of their lived experience. The need for research emerged from the perceived need to increase understanding of 
a commonly used (Baker et al., 2007; Knutzen et al., 2013, 2014), and well accepted (Allison \& Moncrieff, 2014; Mott et al., 2005), yet morally distressing (Larsen \& Terkelsen, 2014) mental health nursing practice. The intervention at times appeared to contradict mental health nursing ethics, yet was simultaneously framed by some researchers as a therapeutic intervention to assist patients in gaining self-control in crisis situations (Currier, 2003; Gonzalez et al., 2013; Larsen \& Terkelsen, 2014; Mott et al., 2005).

The use of a qualitative research approach allowed inquiry into a minimally researched area, increasing understanding of nurses' practices by understanding research participants' subjective experience, laying the foundation for further research. The hermeneutic (interpretive) phenomenology perspective was deemed the best methodology and method to gain insight into the meaning that mental health nurses make of the use of chemical restraint interventions. The interpretive process was used to allow the researcher to describe, analyse, and reflect upon the relationships between the participants and the phenomenon (Converse, 2012; MacKey, 2005; Streubert \& Carperter, 2011). To achieve understanding, the researcher's interpretations went beyond the literal meaning of the participants' words to pursue the fore-structures and thematic meanings held in the data (Mackey, 2005). Methodologically, the back and forth movement between partial and more complete understandings of the phenomenon was conceptualized as a hermeneutic circle (Converse, 2012; MacKey, 2005).

A cursory literature review was conducted prior to analysis to support the necessity of the study, but, in accordance with phenomenological method, was limited to reduce the preconceived notions of the researcher about the phenomenon of inquiry (Fry et al., 2017; Streubert \& Carperter, 2011). Three themes emerged in the literature review: The lack of consistent terminology to describe emergency pharmacological measures to control patients' behaviour crisis, the lack of evidence on clinical practice guidelines, and the ethical challenges of coercive practice in mental health inpatient nursing.

\section{Literature Review}

When this research project began, few peer-reviewed studies were found that specifically focused on chemical restraint, with only one research article found in which researchers specifically used the term 'chemical restraint'. Thus, the initial search was broadened to include search terms related to involuntary medication practices. One central challenge, which emerged in the literature review process, was the inconsistent use of terminology to define pharmacological emergency control measures. Diversity of the definitions used in the articles was important to consider. This literature review begins with an overview of the controversy and varying terminology used to describe "chemical restraint', to highlight the inconsistencies in use of the term in current research.

\section{Definition Challenges}

Much controversy was identified concerning use of the term 'chemical restraint'. Some clinicians viewed the term as outmoded and unethical because of a belief that medication was a therapeutic intervention based on provisional and formal diagnosis (Currier, 2003; Currier \& Allen, 2000). Other clinicians viewed the practice as a coercive, involuntary, emergency measure that posed known risk to the patient (Anderson \& Reeves, 1991; Stastny, 2000). Much research from a physician perspective has focused on the conceptualization of 'as needed' emergency medication interventions as a therapeutic means to control patients' violence in a less invasive way (Currier, 2003; Zeller, 2017). This contrasts with national federal policy treating all types of restraint as equally restrictive (Currier, 2003). Similarly, Bowers, Alexander, Simpson, Ryan, and Carr-Walker (2004) collected evidence supporting the idea of a restraint hierarchy, with chemical restraint viewed as least invasive compared to mechanical and physical restraint. Nursing students gave PRN medication the highest approval rating compared to other types of restraint (Bowers et al., 2007). The researchers' findings supported the perspective 
that chemical restraint was not conceptualized as a restraint, but rather, a therapeutic intervention.

Despite evidence of health care

professionals' different perceptions of emergency psychotropic medication for controlling behavioural emergencies, Currier (2003) found that the distinct term 'chemical restraint' had many negative connotations. Negative views persist despite the potential therapeutic clinical effect for mental health patients (Currier, 2003; Zeller, 2017). Perspectives were physician-based until recent years in which nurse-driven conceptualization and understanding of chemical restraint again highlighted the ambiguity and necessity of comprehensive understanding from a patientcentred and practice-focused perspective (Hu et al., 2019; Muir-Cochrane, 2020; Muir-Cochrane et al., 2019). An exploration of nurses' understandings of chemical restraint interventions helps conceptually clarify the meaning of the term from the perspective of those health professionals who most often administer the intervention.

\section{Chemical Restraint}

Little research was found using the search term "chemical restraint". Rapid tranquilisation is the common term to describe situations of behavioural emergency, requiring fast-acting medication intervention to subdue/control a patient (Allison \& Moncrieff, 2014; Dickinson et al., 2009; Innes \& Sethi, 2013). Research conducted on rapid tranquilisation interventions was focused on etymology or the historical development of the term, clinical practice guidelines, physiological effects, and prescriber indications.

\section{Etymology}

The term 'rapid tranquilisation' was developed as a means of avoiding the use of the term 'chemical restraint' and to shift the focus to the use of rapid-acting medication to treat targeted behaviours in behavioural emergencies (Allison \& Moncrieff, 2014). Chemical restraint is laden with negative connotations, which some argue ignores the therapeutic value of the psychotropic medications on mental illness symptoms. Similarly, some perspectives pathologize violence reframing it as something requiring treatment in the form of rapidly acting sedating medications (Currier, 2003; Zeller, 2017). Arguably this ignores the harms that such interventions cause to the patient, including psychological and physical trauma (Huckshorn, 2006). Historical perspectives have been used to explore the concept of rapid tranquilisation with respect to the development of antipsychotic medication, the origin of the term, the practice of emergency sedation, the historical context, and the views on usage (Allison \& Moncrieff, 2014). Physician-driven research promotes shifts for clinical research to focus on the therapeutic benefit of antipsychotic interventions, rather than maintaining a narrow focus on emergency sedation for chemical restraint only (Allison \& Moncrieff, 2014; Currier, 2003; Wilson et al., 2017; Zeller, 2017). However, a nursing perspective allows for the integration of critical analyses of chemical restraint as a therapeutic intervention, highlighting the potentially significant adverse events that result from the administration of chemical restraint itself and the necessity of clearer conceptualization of chemical restraint (Muir-Cochrane, 2020; MuirCochrane et al., 2019). Although researchers tend to group together all restraints, along with other coercive practices like forced treatment for involuntary patients, it must be kept in mind that forced medication is not necessarily a chemical restraint.

\section{Coercive Practice}

Mental health nurses face daily morally complex ethical challenges (Hem et al., 2014). The inpatient mental health setting requires nurses to exercise power and control, often experienced as coercion by patients, resulting in multiple ethical challenges for nurses (Olofsson \& Jacobsson, 2001). Ethical practice is a central concept in mental health nursing, especially with respect to use of coercive practices like restraint interventions. Most of the literature on ethical practice in mental health has been opinionbased, focused on the involuntary treatment received by patients on acute inpatient mental health units (Stastny, 2000). Researchers have identified the challenges that exist with respect 
to workplace constraints, the importance of autonomy and patient engagement, the negative effects of coercion on therapeutic rapport, principles of patient dignity, and the detrimental effect of coercive practices on staff morale. Nurses are in a challenging position, increasingly marginalized and subject to mandatory administrative work, which detracts from their availability to therapeutically connect with patients and to get to know patients (Rose et al., 2015; Shattell et al., 2008).

Interventions like chemical restraint may be becoming more prevalent because of workplace challenges like staff shortages and increasing incidence of patient violence (Cowin et al., 2003). Interviewing direct-care nurses to gain insight into the nuances of chemical restraint interventions was thought to provide a means to identify changes in a specific coercive practice, as related to the changes in the context of acute inpatient settings. Qualitative exploration of nurses' perceptions of chemical restraint interventions may be beneficial because patients were found to view their inpatient mental health experiences more positively when less coercion was perceived (Sheehan \& Burns, 2011). Deliberate sampling of nurses with diverse levels of experience is necessary to attain differences in perception and understanding.

\section{Theoretical Framework}

To make sense of the data, Goffman's seminal work on the total institution was mobilized. According to Goffman (1961), the total institution is a residential place structured to contain large numbers of individuals with similar living situations in a manner that isolates them from greater society for an extended time period. The controlled structure is determined by those in power and enforced on those with less power. Mental health inpatient facilities are conceptualized as total institutions that contain patients who are deemed unable to care for themselves and who pose an unintended threat to society. Patients and staff are necessarily split because patients live in these institutions, while staff go home at the end of the shift.

\section{The Research Design}

Exploratory inquiry is best suited to fulfill the purpose of research when little formal research evidence is available on the topic. The findings can provide the initial step in laying the foundational groundwork for further inquiry. Phenomenology is both a philosophical perspective and a method. There are different phenomenological schools of thought; the philosophical tradition being followed in this study was originated by Heidegger. One of the major benefits in using hermeneutic phenomenology in the tradition of Martin Heidegger is that researchers can include reflexivity of their influence on their research (Grypdnock, 2006; Streubert \& Carpenter, 2011). The purpose of phenomenological study is to gain insight into the lived experience of a particular phenomenon (Streubert \& Carperter, 2011). The research approach the researcher's role, study setting/participant selection, ethical considerations, data collection, and data analysis were consistent with principles of hermeneutic phenomenology.

\section{Research Method}

Data were collected using semistructured interviews. The qualitative interviews were semi-structured because the data were collected through a goal-driven conversation with sequenced themes to be discussed (Kvale, 1996). The interview method was chosen because of the research goal of understanding nurses' lived daily world from their perspectives. Van Manen (1997) suggested that the researcher maintain a strong orientation to the research question, while using concrete questions focused on specific situations or events. The researcher can explore each experience fully following the identification of the experience by the research participant. Although there is value in creating an interview guide, silence, repetition, or questioning thoughts were identified as effective tools to bring the discourse back to the concrete experience (van Manen, 1997). Open-ended questions can generate rich description by participants about their experiences (Streubert \& Carpenter, 2011). Data were recorded using a 
digital recording device. During the informed consent process each participant was asked for consent to digitally voice record her interview.

\section{The Researcher's Role}

Hermeneutic phenomenology requires that researchers maintain awareness of their personal biases (van Manen, 1997). Explication of the biases was identified through continual self-reflection and the writing and re-writing process; reflection occurred intentionally at every stage of the research process. The researcher is a registered nurse who holds a Canadian Nurse Association Certification in Psychiatric and Mental Health. The researcher currently practices in multiple mental health care clinical settings. The researcher's educational preparation includes a Bachelor of Nursing, Bachelor of Sociology, Bachelor of Psychology, Master of Nursing, and Master of Psychiatric Nursing. Areas of professional practice include inpatient mental health rehabilitation, mental health outreach, and clinical instruction for undergraduate Bachelor of Science in Nursing students. The researcher practices nursing by using a recovery-oriented, trauma-informed perspective. The research was pursued to complete the researcher's Masters thesis. The researcher has no associated conflicts of interest.

\section{Participant Selection}

The study focus was nurses' experience in the use of chemical restraint within adult acute mental health units located in general hospitals in large urban centres located in the Lower Mainland, British Columbia. Participants reportedly worked in care areas providing acute inpatient services primarily to adults 19 to 65 years of age. Nurses who work on acute mental health units in urban general hospitals were chosen because research data supports that patients admitted to such centres are diverse, in terms of varying diagnoses, age range, and inclusion of patients, who had voluntary and involuntary admissions (Canadian Institute for Health Information, 2014).

Purposive snowball sampling was used (Streubert \& Carperter, 2011). Participants targeted for the sample were direct care mental health nurses (either registered nurses or registered psychiatric nurses), with more than one year of experience on inpatient units, and employed permanently (as opposed to casual or temporary) on inpatient units. Nurses with fulltime or part-time employment of more than one year of experience were chosen with the underlying assumption that those nurses will have had greater exposure to chemical restraint interventions. All nurses interviewed were required to have had experience in administering chemical restraint and/or have had direct involvement in team interventions using chemical restraint to be included in the study. Participants were included who recently had worked in acute inpatient psychiatry inpatient, but whose current practice was in a non-acute mental health area.

\section{Participant Description}

There were eight participants, each of whom was interviewed once. All participants were self-identified women, self-reporting their age ranging from 26 to 58 years old. Three participants identified as Registered Nurses, holding undergraduate nursing degrees; five participants identified as Registered Psychiatric Nurses, four holding undergraduate psychiatric nursing degrees and one holding a psychiatric nursing diploma. The participant group was diverse, in terms of work experience in different health organizations, different hospital sites, and on different acute units. The number of years of experience of each nurse ranged from 1.5 to 9 years.

\section{Data Collection}

Ethical principles must be upheld at every stage of the research process (Streubert \& Carpenter, 2011). Ethical considerations include discussion of informed consent, confidentiality and anonymity, as well as benefits and risks for study participants. Research ethics review board approval was obtained from the University Research Ethics Committee. Interviews were recorded using digital voice recording. The interviews ranged from 32 minutes to 98 minutes in duration. Interviews were conducted in a private location chosen by each participant. 


\section{Data Analysis - Emergent Themes}

The overarching themes that emerged in the original data analysis process were as follows: 'Using all the tools in the toolbox,' 'taking control to maintain safety,' 'using therapeutic intervention,' 'working within constraints,' 'making medication choices,' and 'transitioning from novice to expert.' The focus of this paper is on two of these themes that best illustrated the complexity of the ethical issues nurses encounter when choosing chemical restraint interventions: 'taking control to maintain safety,' and 'working within constraints.' The tension between patient safety and patient coercion is used to illuminate and challenge common discourse of person-centred care and recovery-oriented approaches within a culture that frequently uses the coercive practice of chemical restraint. Taking control to maintain safety was divided into the following six subthemes: 'managing the milieu,' 'managing risk,' 'managing fear,' 'managing coworkers,' 'managing the patients/patient behaviours,' and 'using a language of control.' Working within constraints included three subthemes: 'staffing challenges,' 'environmental challenges,' and 'limitations of knowledge/skills.' Exploration of the subthemes within the two specified themes will be used to identify the ethical dilemmas and practice challenges the research participants faced in a complex care environment.

\section{Taking Control to Maintain Safety}

The notion of using medication to take control was discussed by all participants as a means of maintaining patient and staff safety on the inpatient acute mental health unit. Control was distinctly discussed as a means for nurses managing the unit milieu, managing risk, managing fear, managing coworkers, and managing patients/patient behaviours.

Participants also used unique language that conveyed exercising their control. As described in the following quote, when medication was deemed necessary for control purposes the use of the medication was no longer negotiable with the patient.

A lot of times people do refuse to take all their medications. Sometimes they only end up taking them because they've been informed it's not really negotiable and if they don't take it or [if they try to] leave they will get an injection of it so it's kind of a coerced cooperation with them. (Participant 3)

The underlying theme connecting the different areas of control was the emphasis placed on using medication, described in a coerced way, to help manage situations of behavioural emergency to maintain patient and staff safety.

\section{Managing the Milieu}

Chemical restraint was used by participants as a means of managing the milieu (or atmosphere) of their inpatient mental health units. The descriptions of the inpatient units included the words, "chaos," (Participant 4) and "unsettled," (Participant 3) with the choice to use PRN medications sometimes being dependent on the impact of behaviours of one patient on the other patients admitted to the unit. The term "bad," (Participant 5) was used to describe patients who were argumentative, or who did not get along with the other patients admitted to the unit, indicating value judgements essentializing the patients as their behaviours. The nature of the acute inpatient unit was described as busy, with nurses sometimes feeling overwhelmed, turning to the use of chemical restraint medication as a means of keeping the unit calm and controlled as demonstrated in the following quotes.

Some days it can be fine, and other days it's just one incident after another, you know depending who is on the unit and how acute everybody is. We get everybody settled, they're all ready to go, then the nice unit is calm and nice... But when they are all gone and we get a bunch of new people then it is just chaos for a while... and they triggered each other...we don't want it to get to that point, but we watch it, give PRNs to keep them calm before that happens right? (Participant 4)

Depending on the milieu of the unit, sometimes [emergency injections are] happening twice a day and then 
sometimes it does not happen for two weeks...It just kind of depends on if we get a bad group of patients. Especially ones that are, like, when patients are more argumentative with each other and they are not getting along.

Especially...young males...we find that if they are arguing. Getting into our unit...we can end up doing a lot more [chemical restraint]. (Participant 5)

I think there is always the hope that you can spend a lot more time with your patient and really be able to have a therapeutic relationship and have a conversation that is more effective than giving pills. But I think about how sometimes the milieu of the unit just does not allow you to do that. You have your other patients that you need to look after, so it's kind of a little bit easier to give, unfortunately for lack of a better word, easier to give them, and then just try to work on your other patients as well too. Because, being in an acute care unit, there is just so much going on all the time. (Participant 7)

\section{Managing Risk}

Risk assessment was discussed as an integral part of the decision-making process to use PRN medication to control certain situations. Formal risk assessment tools were not identified; however, participants identified characteristics of different risks presented to both themselves and their patients when a behavioural crisis was occurring and chemical restraints considered; for example, violence, self-harm, and medication side effects. Participants identified the necessity of managing risks to the patient, in terms of trauma experienced by the patient requiring chemical restraint and the risk of medication side effects. Participants also noted the risk posed to staff from the behaviours of patients requiring emergency medication in situations of behavioural crisis. The risks were sometimes considered a decision between causing harm to the individual patient and the harms that were posed to staff and other patients by not controlling the situation through use of chemical restraint.

A lot of times it can be tough to administer the medications. I think it would be IM medications especially, it can be...traumatizing for the patients because they're held down a lot of the time if they don't take it orally. You don't want to have an injection, so they have to be held down by security, and that's, and that's terrible...it's hard to do that but I mean, you have to. (Participant 1)

Sometimes we can see, we are really struggling with a patient, and the patient does not have enough medication. And because they do not have enough medication...the staff feels that they are put at risk. The patients are put at risk, and even that patient is being put at risk, because a lot of the time if they are not medicated, a lot of the time they are verbally and physically violent. And because they are like that they end up in a security room, and really the only way to get them out initially is medication. Until they settle down and then we can take them out. (Participant 5)

Some participants identified the constant calculation that they had to make in terms of risks of staff injury and benefits of entering situations where rapid tranquilisation was ordered.

There's just one [psychiatrist] who has a habit of...prescribing ridiculous baby doses of medications that put the team in greater harm's way then...the benefit does not outweigh our safety risk.

(Participant 6)

Two participants identified concerns about risks posed to the patient by taking the medication, in terms of side effects experienced, and causing the patient to feel disconnected from their body, respectively.

I don't take meds because there are side effects to all meds... And that's the unfortunate part of it, and these people 
are on long-term medication and they've got...metabolic syndrome and all the other side effects that go along with it. I mean, I don't blame them for not being for being non-compliant with their meds when they're out in the community...It's a double-edged sword, right...I mean I see them getting better, I do see their minds clear as best as they can and you know? (Participant 4)

I was talking to some of the students yesterday in the class that I was teaching...some of them actually said that they would opt to be locked in a room rather than chemically restrained because they don't have control over their body and I've never actually really thought about that piece before...working in an acute setting I have always thought that chemical restraint is fair, like it's least restraint, in some ways. And I think, if I was in that situation I would probably just want to have a pill rather than be locked in a room but I guess that's not for everybody...I did not really think about that before like how awful it might feel or the side effects... (Participant 7)

Risk was something that participants faced daily, commonplace in the acute mental health nursing workplace. Legal risk was not mentioned, as the focus was on immediate physical harms. With the risk sometimes came fear, which was found to be another important factor to be controlled in order to provide safe patient care.

\section{Managing Fear}

Participants identified that their own fear was a factor in choosing a more invasive medication delivery method. The fear often was described as greater when working with patients who were not known to the nurse. Fear was identified as fear of the unknown, sometimes fueled by stories or situations that they knew about, which were related to co-workers.

I think in some situations, I get scared so you kind of go for the IM faster, and you don't really sit down and talk with the patient. I find if I know the patient well and I know that they are not going to hurt me or anything like that I will sit down with them and give them more time to take the pill rather than an injection. But I think there is always that fear of the unknown with somebody... I have not seen the staff like be physically punched or anything like that but, one of my co-nurses from [the unit], I was not there the day that this happened but she did get attacked, so I think there is that vicarious trauma piece. (Participant 7)

Fear was identified as a factor that influenced nurses' decisions to use more invasive interventions, such as intramuscular injections rather than oral medications. Fear was the result of both knowledge of previous situations that they had observed or heard about, and from distinct types of behaviours that they had observed that led them to believe that the outcome would result in violence or situations of elevated risk. The fear response led to nurses taking the initiative in managing different patient behaviours through use of medication.

\section{Managing the Patient/Patient Behaviours}

Control was identified as needing to be exerted by nurses to manage patients by managing distinct types of patient behaviours. The patient requiring chemical restraint was described as angry, agitated, disorganized, and aggressive. Common worrisome patient behaviours identified were swearing, pacing, suicidal acts, and violent acts (verbal and physical).

There was one guy who was, he was really mad. I guess his belongings were lost somewhere between the police bringing him into emergency. So, we didn't have his belongings, and he escalated...So, I got out the loxapine PRN and was going to give it to him and he kind of, was like, "no I don't need that," and then I think I just gave him a little bit of space and told him, "you know...you are clearly in distress and if you're in distress and you're agitated then that's going to end up with a seclusion... So, it would be good to take the medication, to try to help ease your 
anxiety and calm you down because it's just not going to end well, you know?"...he took it. And that happens, pretty commonly people take it, but if it doesn't then you have to do the IM. (Participant 1)

They also misinterpret things and they get mad because they are there or you know because they are brain-injured and they have poor impulse control and...it's just the nature of who they are. The antisocials they think none of the rules apply to them, I'm just you know, you give me what I want or I'm used to intimidating by bullying people, I'm used to getting my needs met that way so I am going to try to pull it on you guys, right? (Participant 4)

Those nurses with more years of experience used words such as, "threaten" to describe some of the coercive practices that they used to control patient behaviours. Terms such as "antisocials" (Participant 4) demonstrated expectation of certain behaviours from those labelled with a certain diagnosis. Sometimes participants showed a reluctance to use phrases that conveyed violence towards patients as highlighted in the following response:

You would do your best to explain to them why you are giving the medications as well as why they need it... But if they are still acutely agitated, and not calming down, then, I hate to use the word, sort of, threaten them... I would, remind them that...taking the medication will help settle them, but if they don't take it by the oral route then you will have to suggest taking it by injection. (Participant 2)

Participants experienced their use of chemical restraint interventions to control patients as a necessary part of their job because it allowed them to control situations that were escalating or had the potential of escalating.

\section{Using a Language of Control}

Participants used language that was demonstrative of control, a lexicon shared amongst participants despite working in different work-sites and having different years of nursing experience. Terms that indicated a sense of forced control, "take down," and "bring them down," were used to describe the situation of holding a patient to administer intramuscular injection. The terms "snowed," and "knocked out," were used to describe the situation of giving patients medication that heavily or overly sedated them, indicating increased control of potentially serious situations. There was some judgement of patients who sought out PRN medication and were identified as "medication seeking". They were stereotyped as people with potential addictions issues with control being exerted to refrain from using medications because they would not be as effective due to high incidence of drug use before admission and/or because medication really was not needed.

If they're labelled...oh, they're "addicts," if they're, "addicts"...perhaps certain nurses, if a patient is asking for a PRN, the nurse will say "no" because they don't actually need it and they're, "just addicts med seeking". But then you'll also have a nurse, maybe who has a patient who asks, or who doesn't ask, for a medication and then that nurse really wants them to take the medication. (Participant 2)

Medication interventions were frequently described as a means of control. However, participants also discussed the place of medications as a means of providing therapeutic interventions to patients experiencing distressing situations.

\section{Working Within Constraints}

Multiple workplace constraints were identified that influenced use of chemical restraint, including staffing challenges, environmental challenges, and limitations in knowledge. 


\section{Staffing Challenges}

The decision of when to use chemical restraint interventions was impacted by various team and staff factors. Use of chemical restraints often was described as a team response because of the safety risk posed to staff and other patients by the patient identified as experiencing behavioural crisis. The impact of a teamoriented approach can be quite profound in either highlighting interpersonal problems that exist within the team or reinforcing the cohesiveness of the team.

When it's working well those types of situations and interventions can sort of reinforce that the team is working well. When the team is not working well, it reinforces that the team is not working well. Individuals will, sometimes will clearly not be on the same page when something is happening, will disagree that the intervention was done at all. And I would say, more often than not, it's usually talked about in a professional manner...I have not personally been involved, but I have seen other colleagues very nasty towards each other. (Participant 3)

There's always a nurse that you don't want to work with because they don't PRN their patients and then their patients run rampant so to speak, whipping up others and agitating others and needed a, practically a constant on the unit, when they just could have been PRNed...in a positive way...it impacts relationships in that you want to work with people who are actually watching their patients and making note of their mental status properly and if they need some help...that's always a better shift than if you are going to work with people who are negligent in that way. Then again, I used to work with a nurse... she came onto shift and she flat out said, "I'm going to snow all my patients". But you haven't met them yet...That's alarming. (Participant 6)

\section{Environmental Challenges}

Chemical restraint medication was identified as a strategy used to overcome environmental challenges of older, poorly designed, overcrowded, or overly restrictive patient care areas. As described by Participant 3, "there's nothing I can do about the physical layout of any unit," meaning that the only viable choice was to adapt by using medication as a means of controlling patients in environments not designed for acute mental health patients. Further elaboration on the topic was provided:

They are really crowded. They were never designed for Psychiatry to begin with. There's only one eating area. There's one television area; it's loud. There's lots of stimulation. If you were feeling really agitated there's nowhere you can go that's quiet to calm down. ...If you already are agitated that environment is not going to help...the actual seclusion room area... it's very dark, it's like a prison. I can't describe it any other way. It's like a prison... You feel disoriented, just working there. I cannot imagine being in that area as a patient for more than a few days. The seclusion rooms are like prison cell blocks ... So there's a lot of medication given in there just because physically if they had a better set up they would be able to calm themselves a little bit easier. (Participant 3)

They have the TV room...it's super tiny. And basically it just fits, maybe six seats, and it's one TV and one remote with 12 patients, who have nothing else to do so...they're not allowed anything...crayons, they can have crayons, and they can have some magazines, and that's it. Like, there's literally nothing else to do...so, really they're stuck there, for weeks and there's nothing to do. So then they just get into fights with others patients. (Participant 5) 
Participants clearly identified factors that they believed could promote a decrease in the use of chemical restraint practices.

All the patients here have private rooms.

So they have their own private space.

There's also...common areas with

TVs... like a lot of resources for the patients to be able to...calm down, or spend alone time. Which I think is very helpful, versus if you're on a ward where you've got overcrowding and you've got 15 people fighting over one TV. Like if you have one elevated patient who's really needs some quiet space but you really don't have that room, I think that that can be disastrous. So, yeah, the fact that the layout of here, I think is really therapeutic for, for our patients. It really helps. (Participant 2)

The unit that they are on, everyone has their own room...there are two separate $\mathrm{TV}$ areas that are at opposite ends of the unit, um, so that if you are having conflicts with someone you have the option of going somewhere else...two separate dining areas, so again, if you are having difficulties with a particular individual, you can move to a different area to eat. There is an outdoor balcony, so it is a locked unit, but there is an outdoor balcony that you can go out on and have fresh air at any time. You....are not limited by pass privileges. That makes a huge difference a lot of times when people are getting agitated, they can just go to their own room and calm down on their own. (Participant 3)

Identification of design changes that could be made was anecdotal. Some ideas for change were voiced by participants who had worked in different care areas where they could compare the physical design of the care area in connection to between-patient conflicts, and access to outdoor space and private quiet spaces.

\section{Knowledge Limitations}

No participants identified formal training that they had received in the use of chemical restraint interventions. When specifically asked about where their knowledge came from, all participants stated that it was from watching other nurses. Learning happened on the job, through observation and mentorship from more experienced nurses, rather than in formal classroom training.

You kind of learn as you go, but mainly I learned in my preceptorship. My preceptor taught me about all the different PRNs and, also speaking with the... psychiatrist and the pharmacist as well... Because some psychiatrists prefer you to use different...Ativan and loxapine are like the go-to usually. But sometimes if that's not effective for people, or if they, if the psychiatrist wants to try something else, then they'll let you know and they kind of teach you about it...I feel like I'm still learning, but I started to. (Participant 1)

I'm still such a new nurse, I feel like most of my learning has actually been done from other nurses. And when I like their practice, and they have really good rapport with their patients, I just make note. And I just try and have a lot of conversations, just passing it on to the others. You know, like, "yesterday I gave 25 to so and so and it snowed them, and I did not mean to do that, so if you have to give him something today I just recommend giving less." Things like that. But yeah, just taking note of, I would say, more experienced nurses, and how their practice is, and what they do. I can't think of a more, like more formal education than that. (Participant 6)

In addition to lack of formal training, some participants identified the experiential learning limitations that they had in their undergraduate nursing preparation.

My mental health rotation was on the unit I started working on. My first mental health rotation...in a clinical...setting was actually in my 
preceptorship... so had I not done my preceptorship on [an acute mental health unit] I would have gone through nursing school without doing any clinical... Over $50 \%$ of my graduating class did not actually get a mental health rotation. (Participant 2)

There was a lack of formal training that involved best practice with respect to the current evidence on chemical restraint practice, with some reliance placed on the knowledge of psychiatrists to inform nurses about medication practices, as discussed by Participant 4: "They are more aware of all the side effects than we are, of all the long-term side effects...so they are mindful of that, too." However, after medication orders were written by the physicians, the decision about particular medications used was in the hands of the nurses, highlighting the importance of understanding and addressing the knowledge gap.

\section{Discussion}

The two themes identified along with their subthemes highlighted the complexity of ethical issues of using chemical restraint: taking control to maintain safety, and working within constraints. Findings demonstrate the integral ways nurses make meaning of the taken-forgranted common practice of chemical restraint, foregrounding the complex clinical and ethical decision-making processes involved in psychiatric nursing care. The tension between patient safety and patient coercion illuminates the challenge mental health nurses face in providing person-centred and therapeutic care within workplace environments prioritizing control through coercion and safety while posing potential iatrogenic risk. Discussion focuses on challenging cultures that condone frequent use of chemical restraint practices despite lack of clear guidelines and training. The discussion is divided into four subsections: defining chemical restraint, ethical use of chemical restraint within workplace constraints, supporting medication best practice, and education recommendations. Consistencies and differences between current literature and the findings of this study are identified, highlighting the contributions that this research lends to the extant knowledge base. The implications of this research for nursing practice and policy are included. Suggestions for education are provided. Lastly, strengths and limitations of this study are examined.

\section{Defining Chemical Restraint}

The term 'chemical restraint' is problematic because of multiple understandings in practical meaning (Hu et al., 2019; MuirCochrane, 2020; Zeller, 2017). The term was not common knowledge with differing understanding of practical applications, consistent with a medical perspective in which violence is pathologized and treated with medication (Paton et al., 2019; Zeller, 2017). From a nursing perspective medication ordered for chemical restraint was perceived as having more applications than subduing or sedating a patient within the context of acute behavioural emergency, indicating the need to redefine, or clearly define the term chemical restraint for inpatient mental health practice. The term, 'rapid tranquilisation,' was an unfamiliar term, despite it's use in the research literature to describe IM administration of chemical restraints (Allison \& Moncrieff, 2014; Dickinson et al., 2009; Innes \& Sethi, 2013; Paton et al., 2019), indicating a knowledge-practice gap, even for those nurses who indicated research-guided practice. These findings support that conceptual clarity is required to disentangle clinical indications, but also, importantly, what a chemical restraint intervention means to the patient being restrained (Muir-Cochrane, 2020). This is new evidence of nurses experiencing ambiguity of the concept of chemical restraint, blurring boundaries between therapeutic intervention and safety control measure. Consistent, standard terminology can support nurses' common understanding of chemical restraint (MuirCochrane, 2020).

Ambiguous terminology must be resolved to better support patient safety, substantiating use of chemical restraint as a last resort measure, meaning that patient and staff safety outweighs the risk of harm of the intervention of the patient (Hu et al., 2019; Muir-Cochrane, 2020). A novel finding of this research is that the language used to discuss 
chemical restraint events was noted as paternalistic, controlling, and conveying violence used towards patients. The language and terminology used to describe chemical restraint interventions illustrates the impact and influence of language on nursing practice, uncovering the tension between the need to control and the desire to uphold the ethical principles of nonmaleficence and autonomy. The effect of changing language in mental health care can be profound in reducing stigma and creating a culture of peace (Alex et al., 2013; Hamilton \& Manias, 2006). Development and consistent use of a common language that provides objective definition of chemical restraint and rapid tranquilisation practices, from a place of support rather than control, will help nurses foster a therapeutic culture.

\section{Dichotomy of Help and Harm}

Nurses held a dichotomous view of chemical restraint medications. Chemical restraint medications were considered safer than the alternatives of seclusion and mechanical restraint, and an ethical practice intended to prevent greater harms, but caused moral distress when used coercively or invasively, particularly with forced intramuscular injection inconsistent, with research identifying overarching general discomfort in using restraint (Bigwood \& Crowe, 2008). The level of moral distress perhaps was connected to the lack of clear policy, education, and formal guidance for best practice. Findings are consistent with research that supports nurse education in the clinical decision-making process to help nurses identify early signs of escalation and to promote deescalation strategies (Eskandari et al., 2018) to potentially include the use of oral medications. Critical evaluation is necessary prior to inclusion of practice standards that formalize the commonly understood but unresearched 'hierarchy of invasiveness', in which oral medications are used prior to invasive injections of medication (Atkinson \& Garner, 2002).

Chemical restraint was beneficial for controlling quickly escalating behavioural emergencies but also caused participants' moral distress, trauma to nurses and patients, and anger and resentment of patients towards nurses consistent with existing evidence on restraint interventions (Muir-Cochrane et al., 2019). Nurses need explicit education on potential physical and psychological risks posed to both patients and staff members when a more invasive intervention such as rapid tranquillisation is administered. Nurses must be informed of the potential benefits of a less invasive intervention, such as oral chemical restraint. Findings highlight the importance of identifying and naming coercive practices and the power differentials nurses experience with their patients, including considerations about the many workplace constraints that also influence nurses' decisions to use chemical restraint interventions.

\section{Ethical Use of Chemical Restraint within Workplace Constraints}

Nurses work within a myriad of workplace constraints that influence their practice (Bigwood \& Crowe, 2008; Shin et al., 2018). Workplace limitations were identified that resulted in chemical restraint to manage risk, take control, and assert power in behavioural crises. Findings provided some indication of specific circumstances and influences on nurses' choice to use chemical restraint. In the following sections, challenges of the workplace environment, therapeutic use of medication ordered for chemical restraint, fear of the unknown, and the role of coercive medication practices are addressed.

\section{Challenges of the Workplace Environment}

Incidents were identified where chemical restraints could have been avoided if the workplace environment was better designed and better resources were available to support non-medication interventions. Participants consistently identified multiple workplace challenges that led to increased chemical restraint use. Chemical restraint intervention was sometimes considered the only viable option to manage risks when caring for multiple patients experiencing multiple stressors, including substance cravings and acute mental illness symptoms of paranoia, boredom, lack of private space, and living amongst many angry and bored co-patients. The results echo the findings that 
specific interventions, such as chemical restraint, increase in prevalence due to multiple workplace challenges, including staff shortages and increasing incidence of patient violence (Cowin et al., 2003; Shahpesandy et al., 2015). Chemical restraint best practices must be designed to emphasize safe patient care, as well as to ameliorate the challenges posed by shortages of staffing, crowded units, and poor inpatient mental health unit design. Promoting early interventions from a policy and leadership level may further decrease use of chemical restraint. Including the patient input in choice of PRN psychotropic medication for symptom treatment and management, rather than emergency chemical restraint to sedate and subdue, may also prove beneficial.

\section{Fear of the Unknown}

Participants disclosed their fears about providing care for new patients because of the potential for unpredictable behaviours resulting in violence. The subsequent fear contributed to the use of chemical restraint. Participants were more comfortable with patients for whom they previously had provided care; patients with whom they had established therapeutic rapport and held some predictability of behaviours, because of known patient history. Efforts were made to plan care using information available about patients' histories, demonstrating participants attempted to include patients' preferences in the intervention planning processes in the event of behavioural emergencies. However, patient care information was conferred from the nurse's perspective, with little mention of discussion and debriefing with patients to create long-term aggression or crisis management care plans. To improve personcentred and trauma-informed practice, crisis management care plans could be created and contained as part of the permanent record, with copies given to the patient and family, to be presented in the event of acute inpatient admission. More inclusion of patients' families and community providers may be helpful in reducing coercive practices.

\section{The Role of Coercive Medication Practices}

Coercive medication practices are a mainstay for acute mental health nursing, with the resulting moral distress an accepted aspect of the job. Power differentials between patients and nurses were prevalent, especially for patients certified under the British Columbia Mental Health Act, perpetuated by participants' understanding of acceptable forced treatment in accordance with medication compliance by any means necessary (Gray, Hastings, Love, \& O'Reilly, 2016). Though acknowledged, few suggestions were made to alleviate coercive practice. The lack of readily accessible or accessed alternatives may indicate the influence of the mental health system as a total institution, as described by Goffman (1961).

The overarching culture and practices of the hospital deeply influence staff's shared understanding of the ascribed meaning of certain patient behaviours labelled dangerous and violent, necessitating chemical restraint interventions. The result is that the potential difference in meaning that patients ascribe to certain behaviours, as those nurses described with words like "antisocial," "agitated," "aggressive," may be erased or ignored in the name of safety achieved through control.

\section{'Patient' Perspectives.}

Similar to the findings of MerinaeauCote et al. (2014), participants discussed the emotional distress that they experienced in administering medications under circumstances of coercion. However, reducing coercive practice is essential to build patient trust and thus reduce the frequency of incidents that require chemical restraint interventions (Gilburt et al., 2008), which benefits the patients and reduces the nurses' moral distress. Strategies identified to reduce use of forced medication were: administering medications early, asking patients about preferences, listening for explicit requests for medications, and honouring patients' stated preference for oral or injection medication when in crisis. Engaging with patients early in their admission, to identify their preferences and intervening at the first signs of distress, was a key strategy to assist staff 
members and reduce coercive practice and moral distress related to use of forced medication interventions. Research findings support the inclusion of patients in care planning decisions to strengthen the therapeutic relationship between nurse and patient and decrease patient perception of coercion (Sheehan \& Burns, 2011). However, the higher the acuity of patient, the more difficulties participants faced when trying to include patients in making care decisions.

Participants identified a lack of integration of patient involvement in care planning when providing care for patients experiencing acute symptoms of psychosis. For example, patients who were experiencing distress related to severe paranoia and perceptual disturbances were described as quickly treated with medications and either transferred to less acute hospital units or discharged soon after symptom stabilization, limiting opportunity to provide input in the decision-making process. Participants working with the most symptomatic patients focused efforts on patient compliance with medication regimes to manage symptoms and reduce risk in the short term, consistent with thought on the risk reduction goal of inpatient acute mental health care (Slemon et al., 2017). Novel findings of this study were the reported lack of thought about the ramifications of chemical restraint practices on patients' lives post-discharge, because focus was on addressing acute concerns limited to the acute inpatient stay. Coercive practices could be decreased by implementing strategies to increase nurses' understanding of the lasting effects that invasive acute inpatient interventions can have on patients; for example, trauma caused by rapid tranquilisation. Increased knowledge about best practice for medication utilization also may have a positive effect on reducing coercive medication practices.

\section{Supporting Medication Best Practices}

Participants reported inadequate knowledge of evidenced-based chemical restraint practices substantiated in their descriptions of practices contradictory to research evidence. The current study findings are consistent with those of previous researchers who claimed that mental health nurses' medication practices and choices often were based on unit culture (Brown et al., 2010) and tradition (Stewart et al., 2012). Few descriptions were provided of the negative impacts of chemical restraint. Participants identified some negative outcomes, including some medication side-effects. At times, knowledge of potential side-effects influenced the decisions to use chemical restraints, especially for those patients with multiple medications orders.

Though participants did identify some of the alternatives to chemical restraint, such as more space and access to distraction activities, alternatives were difficult to implement, especially in higher acuity settings, because of higher security and safety measures.

Participants' perceptions of limited options within a risk averse culture of mental health nursing, led by fear and need to control, may be best addressed by creating a cultural shift away from control and risk management towards patient engagement and relational practice (Slemon et al., 2017). A unique finding is the reported reliance of nurses on physicians for directives in medication administration, which indicates a knowledge gap of nurses who administer the medications, highlighting a need for further exploration on the driver of this gap. Does it exist because of a nursing focus on recovery-oriented models in which medicallyfocused treatments like medications are considered the domain of physicians; or, is it because of hierarchical institution in which physicians retain a stronghold on power through maintaining control of medication knowledge with nurses acting as extensions of the physicians power? At a practical level nurses are the clinicians who must safely assess the patient, administer the medication, and complete the post-intervention assessments; thus, they must take responsibility to learn about the prescribed medications that they are administering to ensure consistent, safe, ethical, and evidencebased practice.

\section{Education Recommendations}

Participants lacked formal education on safe use of chemical restraint. Clinical decisionmaking was developed through role modelling, 
by emulating care that they believed was ethical and competent, and by rejecting care typified as unsafe, unethical, and harmful practice. In this sample, graduates of Bachelor of Nursing, Bachelor of Psychiatric Nursing, and Psychiatric Nursing diploma programs identified limited formal education on chemical restraint practices when students, and they reported no recollection of receiving such education following their entry to practice. Participants relied on psychiatrists for assisting in their clinical judgement about medication choice and dose and their assessment of pre- and post-medication administration (especially in terms of side-effects). They lacked agency in decision-making earlier on in their careers, lacking confidence in openly questioning co-workers' clinical decisionmaking processes and curtailing dangerous practices consistent with research on identity formation in the novice mental health nurse (Hurley \& Lakeman, 2011). Given the complexity of clinical decision-making and the depth of clinical judgment required to provide the intervention safely and ethically, clinical leaders would best serve the interests of patients and clinical staff in the acute inpatient mental health setting by helping nurses develop competencies in the use of chemical restraints earlier in their careers. Findings highlight the value in establishing competencies of chemical restraint starting at the undergraduate level, when nursing students become exposed to psychotropic medication practices. Demystifying chemical restraint practices will allow for the development of confidence in clinical decision making of control measures in behavioural emergencies, encouraging nurses to reflect on and question the ethics of such practices, rather than foster unquestioning acceptance.

\section{Nursing Mentorship}

Education is a key factor, which can be used to improve safety in the use of restraint interventions and reduce the use of restraint practices (Mann-Poll et al., 2013), to develop confidence and reduce fear (Hurley \& Lakeman, 2011). More clinical support is required to support novice nurses in their transition into the workplace. Findings highlight the important role experienced nurses play in informal education of novice nurses. However, those experienced nurses' knowledge base may be reliant on tradition, embedded in the unit culture, and deeply influenced by the constraints of the environment (Alzayyat, 2014). Participants reported lacking confidence in challenging what they saw as unsafe and unethical practices when they were new nursing graduates, unsure if their perspectives were idealistic and unrealistic, in fear of challenging the status quo. Formalized team training focused on practical implementation of best practice specific to a care area may help shift unit culture. Through education of an entire team rather than focusing on individuals, workplace-specific education may be used to address safe and ethical practices given different constraints.

\section{Definitions of Chemical Restraint}

The term, "rapid tranquilisation," was not common knowledge, yet the term is used in the research literature to describe IM administration of chemical restraint (Allison \& Moncrieff, 2014; Dickinson et al., 2009; Innes \& Sethi, 2013). Nursing educators must integrate a standard terminology when referring to chemical restraint interventions to ensure that nurses have the basic knowledge to competently provide these interventions. Curriculum development on the use of chemical restraint also must include definitions of chemical restraint, to help nurses differentiate between PRN medication ordered for specific symptom management and those medications ordered for chemical restraint. Appropriate education with clear definitions of chemical restraint and explanation of practical and ethical implications of different types of coercive nursing practice will support reduction in the use of coercive practice.

\section{Reducing Coercion}

Participants differentiated between worst-case scenarios and those situations that were considered to be common, everyday practice. Oral psychotropic medications were described as given frequently; so much so, that many participants described experiences blending together without salient memories of specific incidents where injection medication 
was required. Nurses require formal education to support integration of recovery and traumainformed practice to support patients and families. Focus also must include strategies to uncover and reduce stigma of patients and fear of patient behaviours such as violence, leaving space for nurses to openly acknowledge the impacts on choice of intervention, the number and types of interventions that are used, and the timing of the interventions.

In theory, the most acceptable reason to use a patient-containment method is violent behavior (Cowin et al., 2003); however, in practice, different behaviours lead to Patient Control Measures (PCMs), including milieu management of the inpatient nursing ward. Education must include nursing interventions necessary for each specific area of practice, with simulation-type practice to increase the awareness of staff about the different constraints that occur in practice. Team factors contribute to a positive or negative workplace. Therefore, policies and programs, as well as education designed to foster positive workplace culture and teamwork, are key considerations.

\section{Promoting a Recovery-Oriented Practice}

Benefit in using a recovery approach has been demonstrated in psychiatric settings (Lim et al., 2017). Recovery-oriented practice involves collaboration between health care provider and patient, supporting the patient through building on his/her strengths, recognizing the importance of engaging the patient, and working with him/her to improve his/her quality of life (Lim et al., 2017).

Integrating a recovery approach often requires a cultural shift due to the pervasiveness of traditional paternalistic medical models commonly used in psychiatry (Repique et al., 2016).

Participants reported efforts to include patients in care planning and to educate patients on PRN use, contrasting with the research of Clearly et al. (2012), where patients admitted to acute inpatient units reported not receiving relevant facts about PRN use. According to participants, patients stated that they recognized the therapeutic value of chemical restraint. Positive experiences were discussed, as patients voiced positive feedback, sometimes thanking nurses for providing an external means of control because of a patient's awareness of being out of control and seeing no alternative means of containment. These findings were in contrast to research in which patients most frequently voiced beliefs of no benefit, nor necessity, for restraint or seclusion interventions (Soininen, et al., 2013), substantiating the benefit of discussing patient preferences of emergency containment methods. Clinical supervision may be a strategy in helping nurses to gain insight into the multiple factors influencing their decisions to use chemical restraint, factors which included fear of the unknown and caring for unfamiliar patients.

Education designed to integrate recovery principles in acute care settings is necessary to shift culture towards collaborative, patient-centered care in which patients are meaningfully included in the clinical decisionmaking process. The recovery approach may be best achieved by bringing together care providers. Use of a multidisciplinary education approach that brings together prescribing psychiatrists and the nurses who administer the medication and provide ongoing care to the patients may prove fruitful in creating mutual understanding and shared practice. Team training may be of benefit to bring together the physicians who are prescribing medications with the direct care nurses and other members of the team.

\section{Strengths and Limitations}

A dearth of nursing-focused research was found on chemical restraint interventions in acute inpatient adult mental health settings, with no Canadian studies found. The major strength of this study is the foundational knowledge of nurses' experiences of chemical restraint utilization in one area of Canada. Use of the hermeneutic (interpretive) phenomenological method, which generated thick, rich description of direct care nurses experiences allowed for deeper understanding of the common mental health nursing practice of chemical intervention, bringing insights into the lived experience of nurses. Findings provided a strong basis for future research nursing practice, ethics, 
education and patient experience of chemical restraint intervention.

Limitations of the study included participants' potential accuracy in recalling past events, participants' potential concern for presentation of self, ambiguity in the use of the term "chemical restraint", and the contextspecific nature of the study. This study was intended to help understand how nurses make meaning of chemical restraint interventions, bringing the taken-for-granted normalized practice into consciousness in a way that can allow it to be understood in the context of mental health nursing practice and mental health practice. The main limitation emerged from potential challenges participants may have had in expressing themselves freely and accurately, as they were recollecting past experiences. Participants acknowledged that occurrences of administering oral medications were more difficult to recall because of the frequency of administering the intervention, resulting in fuzzy memories and more general descriptions. However, in interpretive phenomenology accuracy of memories is not as important as the telling of the experience and the introspective process involved (van Manen, 1997)

Additionally, participants may have been influenced by trying to present a certain positive image of themselves to the researcher. Participants may have been experiencing embarrassment or shame over their roles in these experiences, thus influencing the telling of their story in a more positive light in conversation with the researcher. Similarly, in their recollections, participants decided which medications were ordered for the purpose of chemical restraint, and given the ambiguity of the term, 'chemical restraint' different participants may have interpreted the situations differently.

The purpose of this study was to obtain thick description of mental health nurses' experiences of administering as needed psychotropic medication for the purpose of behavioural emergency. The sample size was small as is common in phenomenological studies. However, participants shared diversity in their unique experiences, despite similarities such as gender, level of education, and geographical location of practice. Useful data were collected and analyzed providing a foundation for continuation of research.

\section{Conclusion}

The author used interpretive phenomenology to gain insight into the experiences of mental health nurses' use of chemical restraint in managing behavioural emergencies in the adult inpatient mental health care setting. The goal of the research was to learn nurses' perspectives to increase understanding of the ways that nurses made meaning of chemical restraint interventions visa-vis the clinical, ethical decision-making process. Insight was provided into the experiences of nurses who regularly used the practice, uncovering the nuances of their lived experiences. The meaning making of eight acute inpatient mental health nurses' experiences of using medication interventions for patients in situations of behavioural crisis allowed thoughtful exploration of the inpatient mental health nurses' challenge of providing therapeutically-minded, safe, and ethical care within multiple workplace constraints. The themes and subthemes may be used as a starting point for additional research on safe chemical restraint practices of acute inpatient mental health nurses to better inform nursing practice and to improve patient care. The implications for improving nursing practice were explored, highlighting significant areas for change. In conclusion, this study provided new insight into the experiences of acute mental health nurses' practices of administering chemical restraints and highlighted gaps in consistent terminology and nursing knowledge. 


\section{References}

Alex, M., Whitty-Rogers, J., \& Panagopoulos, $\mathrm{W}$. (2013). The language of violence in mental health: Shifting the paradigm to the language of peace. Advances in Nursing Science, 36(3), 229-241. https://doi.org/10.1097/ANS.0b013e318 29 edcf3

Allison, L., \& Moncrieff, J. (2014). "Rapid tranquillisation": An historical perspective on its emergence in the context of the development of antipsychotic medications. History of Psychiatry. https://doi.org/10.1177/0957154X13512 573

Alzayyat, A. S. (2014). Barriers to evidencebased practice utilization in psychiatric/mental health nursing. Issues in Mental Health Nursing, 35(2), 134143. https://doi.org/10.3109/01612840.2013. 848385

Anderson, W. H., \& Reeves, K. R. (1991). Chemical restraint: An idea whose time has gone. Administration and Policy in Mental Health, 18, 205-208. https://doi.org/10.1007/BF00713806

Atkinson, J. M., \& Garner, H. C. (2002). Least restrictive alternative - Advance statements and the new mental health legislation. Psychiatric Bulletin, 26(7), 246-247. https://doi.org/10.1192/pb.26.7.246

Baker, J. A., Lovell, K., Harris, N., \& Campbell, M. (2007). Multidisciplinary consensus of best practice for pro re nata (PRN) psychotropic medications within acute mental health settings: A Delphi study. Journal of Psychiatric and Mental Health Nursing, 14(5), 478-484. https://doi.org/10.1111/j.13652850.2007.01112.x

Bigwood, S., \& Crowe, M. (2008). "It's part of the job, but it spoils the job": A phenomenological study of physical restraint: Feature Article. International Journal of Mental Health Nursing, $17(3), 215-222$. https://doi.org/10.1111/j.14470349.2008.00526.x

Bowers, L., Alexander, J., Simpson, A., Ryan, C., \& Carr-Walker, P. (2004). Cultures of psychiatry and the professional socialization process: The case of containment methods for disturbed patients. Nurse Education Today, 24(6), 435-442. https://doi.org/10.1016/j.nedt.2004.04.0 08

Bowers, L., Alexander, J., Simpson, A., Ryan, C., \& Carr-Walker, P. (2007). Student psychiatric nurses' approval of containment measures: Relationship to perception of aggression and attitudes to personality disorder. International Journal of Nursing Studies, 44(3), 349356.

https://doi.org/10.1016/j.ijnurstu.2005.0 3.002

Brown, S., Chhina, N., \& Dye, S. (2010). Use of psychotropic medication in seven English psychiatric intensive care units. Psychiatrist, 34(4), 130-135. https://doi.org/10.1192/pb.bp.108.02376 2

Canadian Institute for Health Information. (2014). Mental Health and Addictions Data and Information Guide, 2014 Standards and Data Submission. CIHI. https://www.cihi.ca/en/access-data-and$\underline{\text { reports }}$

Cannon, M. E., Sprivulis, P., \& McCarthy, J. (2001). Restraint practices in Australasian emergency departments. Australian and New Zealand Journal of Psychiatry, 35(4), 464-467. https://doi.org/10.1046/j.14401614.2001.00925.x

Chieze, M., Hurst, S., Kaiser, S., \& Sentissi, O. (2019). Effects of seclusion and restraint in adult psychiatry: A systematic review. Frontiers in Psychiatry, 10 (July). https://doi.org/10.3389/fpsyt.2019.0049 $\underline{1}$

Cleary, M., Horsfall, J., Jackson, D., O'Hara, Aarons, M., \&Hunt, G. E. (2012). Patients' views and experiences of pro re 
nata medication in acute mental health settings. International Journal of Mental Health Nursing, 21(6), 533-539. doi:10.1111/j.1447-0349.2012.00814.x

Converse, M. (2012). Philosophy of phenomenology: How understanding aids research. Nurse Researcher, 20(1), 28-32. https://doi.org/10.7748/nr2012.09.20.1.2 8.c9305

Cowin, L., Davies, R., Estall, G., Berlin, T., Fitzgerald, M., \& Hoot, S. (2003). Deescalating aggression and violence in the mental health setting. International Journal of Mental Health Nursing, 12(1), 64-73. https://doi.org/10.1046/j.14400979.2003.00270.x

Cunha, M., André, S., Bica, I., Ribeiro, O., Dias, A., \& Andrade, A. (2016). Chemical and Physical Restraint of Patients. Procedia - Social and Behavioral Sciences, 217, 389-399.

https://doi.org/10.1016/j.sbspro.2016.02. 109

Currier, G. W. (2003). The controversy over "chemical restraint" in acute care psychiatry. Journal of Psychiatric Practice, 9(1), 59-70. https://doi.org/10.1097/00131746200301000-00006

Currier, G. W., \& Allen, M. H. (2000). Physical and chemical restraint in the psychiatric emergency service. Psychiatric Services, 51(6), 717-719. https://doi.org/10.1176/appi.ps.51.6.717

Dickinson, T., Ramsdale, S., \& Speight, G. (2009). Managing aggression and violence using rapid tranquillisation. Nursing Standard, 24(7), 40-52.

Emmanuel, L., Taylor L, Hain A, Combes, J., Hatlie, M., Karsh, B., Lau, D., Shalowitz, J., Shaw, T., \& Walton, M. (2013). Module 13d: Mental Health Care: Seclusion and Restraint: When All Else Fails. PSEP-Canada https://www.patientsafetyinstitute.ca/en/ education/PatientSafetyEducationProgra $\mathrm{m} /$ PatientSafetyEducationCurriculum/M entalHealthModules/Pages/Mental-
Health-Care-Seclusion-andRestraint.aspx

Eskandari, F., Abdullah, K. L., Zainal, N. Z., \& Wong, L. P. (2018). The effect of educational intervention on nurses' knowledge, attitude, intention, practice and incidence rate of physical restraint use. Nurse Education in Practice, 32, 52-57. https://doi.org/10.1016/j.nepr.2018.07.0 07

Fry, J., Scammell, J., \& Barker, S. (2017). Drowning in muddied waters or swimming downstream?: A critical analysis of literature reviewing in a phenomenological study through an exploration of the lifeworld, feflexivity and role of the researcher. Indo-Pacific Journal of Phenomenology, 17(1), 1-12. https://doi.org/10.1080/20797222.2017. 1293355

Garriga, M., Pacchiarotti, I., Kasper, S., Zeller, S. L., Allen, M. H., Vázquez, G., Baldacąra, L., San, L., McAllisterWilliams, R. H., Fountoulakis, K. N., Courtet, P., Naber, D., Chan, E. W., Fagiolini, A., Möller, H. J., Grunze, H., Llorca, P. M., Jaffe, R. L., Yatham, L. N., ... Vieta, E. (2016). Assessment and management of agitation in psychiatry: Expert consensus. World Journal of Biological Psychiatry, 17(2), 86-128. https://doi.org/10.3109/15622975.2015. 1132007

Gelkopf, M., Roffe, Z., Behrbalk, P., Melamed, Y., Werbloff, N., \& Bleich, A. (2009). Attitudes, opinions, behaviors, and emotions of the nursing staff toward patient restraint. Issues in Mental Health Nursing. https://doi.org/10.3109/0161284090315 9777

Georgieva, I., Mulder, C. L., \& Wierdsma, A. (2012). Patients' preference and experiences of forced medication and seclusion. Psychiatric Quarterly, 83, 113. https://doi.org/10.1007/s11126-0119178-y

Gilburt, H., Rose, D., \& Slade, M. (2008). The importance of relationships in mental 
The Canadian Journal of Critical Nursing Discourse

See it. Speak it. Write it. Change it.

health care: A qualitative study of service users' experiences of psychiatric hospital admission in the UK. BMC health services research, 8(1), 92.

Goffman, E. (1961). Asylums: Essays on the Social Situation of Mental Patients and Other Inmates. New York: Doubleday \& Company.

Gonzalez, D., Bienroth, M., Curtis, V., Debenham, M., Jones, S., Pitsi, D., \& George, M. (2013). Consensus statement on the use of intramuscular aripiprazole for the rapid control of agitation in bipolar mania and schizophrenia.

Current Medical Research and Opinion, 29(3), 241-250.

https://doi.org/10.1185/03007995.2013. 766591

Gray, J. E., Hastings, T. J., Love, S., \& O’Reilly, R. L. (2016). Clinically significant differences among Canadian mental health acts: 2016. The Canadian Journal of Psychiatry, 61(4), 222-226. DOI: $10.1177 / 0706743716632524$

Grypdonck, M. H. (2006). Qualitative health research in the era of evidence-based practice. Qualitative Health Research, 16(10), 1371-1385. https://doi.org/10.1177/1049732306294 089

Hamilton, B., \& Manias, E. (2006). "She's manipulative and he's right off": A critical analysis of psychiatric nurses' oral and written language in the acute inpatient setting. International Journal of Mental Health Nursing, 15(2), 84-92. https://doi.org/10.1111/j.14470349.2006.00407.x

Hem, M. H., Molewijk, B., \& Pedersen, R. (2014). Ethical challenges in connection with the use of coercion: A focus group study of health care personnel in mental health care. BMC Medical Ethics, 15. https://doi.org/10.1186/1472-6939-1582

Hu, F., Muir-Cochrane, E., Oster, C., \& Gerace, A. (2019). An examination of the incidence and nature of chemical restraint on adult acute psychiatric inpatient units in Adelaide, South
Australia. International Journal of Mental Health Nursing, 28(4), 909-921. https://doi.org/10.1111/inm.12591

Huckshorn, K. A. (2006). Re-designing state mental health policy to prevent the use of seclusion and restraint.

Administration and Policy in Mental Health and Mental Health Services Research, 33, 482-491. https://doi.org/10.1007/s10488-0050011-5

Hui, A., Middleton, H., \& Völlm, B. (2013). Coercive Measures in Forensic Settings: Findings from the Literature. International Journal of Forensic Mental Health, 12(1), 53-67. https://doi.org/10.1080/14999013.2012. 740649

Hurley, J., \& Lakeman, R. (2011). Becoming a psychiatric/mental health nurse in the UK: A qualitative study exploring processes of identity formation. Issues in Mental Health Nursing, 32(12), 745751. https://doi.org/10.3109/01612840.2011. 609634

Innes, J., \& Sethi, F. (2013). Current rapid tranquillisation documents in the UK: A review of the drugs recommended, their routes of administration and clinical parameters influencing their use. Journal of Psychiatric Intensive Care, 9(2), 110-118. https://doi.org/10.1017/S174264641200 026X

Keser Özcan, N., Bilgin, H., Akin, M., \& Badirgali Boyacioğlu, N. E. (2015). Nurses' attitudes towards professional containment methods used in psychiatric wards and perceptions of aggression in Turkey. Journal of Clinical Nursing, 24(19-20), 2881-2889. https://doi.org/10.1111/jocn.12903

Knutzen, M., Bjørkly, S., Eidhammer, G., Lorentzen, S., Mjøsund, N. H., Opjordsmoen, S., ... \& Friis, S. (2013). Mechanical and pharmacological restraints in acute psychiatric wardsWhy and how are they used?. Psychiatry Research, 209(1), 91-97. 
https://doi.org/10.1016/j.psychres.2012. 11.017

Knutzen, M., Bjørkly, S., Eidhammer, G., Lorentzen, S., Mjøsund, N. H., Opjordsmoen, S., Sandvik, L., \& Friis, S. (2014). Characteristics of patients frequently subjected to pharmacological and mechanical restraint- $A$ register study in three Norwegian acute psychiatric wards. Psychiatry Research, 215(1), 127-133. https://doi.org/10.1016/j.psychres.2013. 10.024

Kvale, S. (1996). The interview situation. In Interviews: An Introduction to Qualitative Research Interviewing (pp. 124-143). Thousand Oaks. https://doi.org/10.1037/00223514.90.4.644

Landeweer, E. G. M., Abma, T. A., \& Widdershoven, G. A. M. (2010). The essence of psychiatric nursing: Redefining nurses' identity through moral dialogue about reducing the use of coercion and restraint. Advances in Nursing Science, 33(4), E31-E42. https://doi.org/10.1097/ANS.0b013e318 1fb2ef9

Larsen, I. B., \& Terkelsen, T. B. (2014). Coercion in a locked psychiatric ward: Perspectives of patients and staff. Nursing Ethics, 21(4), 426-436. https://doi.org/10.1177/0969733013503 601

Lim, E., Wynaden, D., \& Heslop, K. (2017). Recovery-focussed care: How it can be utilized to reduce aggression in the acute mental health setting. International Journal of Mental Health Nursing, 10(26), 445-460. https://doi.org/10.1111/inm.12378

Ling, S., Cleverley, K., \& Perivolaris, A. (2015). Understanding mental health service user experiences of restraint through debriefing: A qualitative analysis. Canadian Journal of Psychiatry, 60(9), 386-392. https://doi.org/10.1177/0706743715060 00903
MacKey, S. (2005). Phenomenological nursing research: Methodological insights derived from Heidegger's interpretive phenomenology. International Journal of Nursing Studies, 42(2), 179-186. https://doi.org/10.1016/j.ijnurstu.2004.0 6.011

Mann-Poll, P. S., Smit, A., Van Doeselaar, M., \& Hutschemaekers, G. J. M. (2013). Professionals' attitudes after a seclusion reduction program: Anything changed? Psychiatric Quarterly, 84, 1-10. https://doi.org/10.1007/s11126-0129222-6

McCain, M., \& Kornegay, K. (2005). Behavioral health restraint: The experience and beliefs of seasoned psychiatric nurses.

Journal for Nurses in Staff Development, 21(5), 236-242. https://doi.org/10.1097/00124645200509000-00009

Mérineau-Côté, J., \& Morin, D. (2014). Restraint and seclusion: The perspective of service users and staff members.

Journal of Applied Research in Intellectual Disabilities, 27(5), 447-457. https://doi.org./10.1111/jar.12069

Mott, S., Poole, J., \& Kenrick, M. (2005). Physical and chemical restraints in acute care: Their potential impact on the rehabilitation of older people. International Journal of Nursing Practice, 11(3), 95-101. https://doi.org/10.1111/j.1440172X.2005.00510.x

Muir-Cochrane, E. (2020). A wicked problem: Chemical restraint: Towards a definition. International Journal of Mental Health Nursing, 29(6), 12721274. https://doi.org/10.1111/inm.12780

Muir-Cochrane, E., Oster, C., \& Grimmer, K. (2019). International research into 22 years of use of chemical restraint: An evidence overview. Journal of Evaluation in Clinical Practice, 26(3), 927-956. https://doi.org/10.1111/jep.13232

Muir-Cochrane, E., Oster, C., Gerace, A., Dawson, S., Damarell, R., \& Grimmer, K. (2020). The effectiveness of chemical 
restraint in managing acute agitation and aggression: A systematic review of randomized controlled trials.

International Journal of Mental Health

Nursing, 29(2), 110-126.

https://doi.org/10.1111/inm.12654

Muir-Cochrane, E., Oster, C., \& Grimmer, K. (2020). Interrogating systematic review recommendations for effective chemical restraint. Journal of Evaluation in Clinical Practice, 26(6), 1768-1779. https://doi.org/10.1111/jep.13363

National Consumer and Carer Forum. (2009). Ending seclusion and restraint in Australian mental health services: A position statement by the National Mental Health Consumer \& Carer Forum (NMHCCF), from https://nmhccf.org.au/resources/publicat ions

Olofsson, B., \& Jacobsson, L. (2001). A plea for respect: Involuntarily hospitalized psychiatric patients' narratives about being subjected to coercion. Journal of Psychiatric and Mental Health Nursing, 8(4), 357-366. https://doi.org/10.1046/j.13652850.2001.00404.x

Parker, C. B., Calhoun, A., Wong, A. H., Davidson, L., \& Dike, C. (2020). A call for behavioral emergency response teams in inpatient hospital settings. AMA Journal of Ethics, 22(11), 956964. https://doi.org/10.1001/amajethics.2020. 956

Paton, C., Adams, C. E., Dye, S., Fagan, E., Okocha, C., \& Barnes, T. R. E. (2019). The pharmacological management of acute behavioural disturbance: Data from a clinical audit conducted in UK mental health services. Journal of Psychopharmacology, 33(4), 472-481. https://doi.org/10.1177/0269881118817 170

Perkins, E., Prosser, H., Riley, D., \& Whittington, R. (2012). Physical restraint in a therapeutic setting; a necessary evil? International Journal of Law and Psychiatry, 35(1), 43-49. https://doi.org/10.1016/j.ijlp.2011.11.00 $\underline{8}$

Repique, R. J. R., Vernig, P. M., Lowe, J., Thompson, J. A., \& Yap, T. L. (2016). Implementation of a recovery-oriented training program for psychiatric nurses in the inpatient setting: A mixedmethods hospital quality improvement study. Archives of Psychiatric Nursing, 30(6), 722-728. https://doi.org/10.1016/j.apnu.2016.06.0 03

Registered Nurses' Association of Ontario. (2012). Promoting safety: Alternative approaches to the use of restraints. Registered Nurses'Association of Ontario. https://rnao.ca/bpg/guidelines/promoting -safety-alternative-approaches-userestraints

Rose, D., Evans, J., Laker, C., \& Wykes, T. (2015). Life in acute mental health settings: Experiences and perceptions of service users and nurses. Epidemiology and Psychiatric Sciences, 24(1), 90-96. https://doi.org/10.1017/S204579601300 0693

Shahpesandy, H., Tye, N., Hegarty, A., Czechovska, J., Kwentoh, M. L., \& Wood, A. (2015). Rapid tranquillisation of acutely disturbed and violent patients: A retrospective cohort examination of 24 patients on a psychiatric intensive care unit. Journal of Psychiatric Intensive Care, 11(S1), E1. https://doi.org/10.1017/S174264641500 0072

Shattell, M. M., Andes, M., \& Thomas, S. P. (2008). How patients and nurses experience the acute care psychiatric environment. Nursing Inquiry, 15(3), 242-250. https://doi.org/10.1111/j.14401800.2008.00397.x

Sheehan, K. A., \& Burns, T. (2011). Perceived coercion and the therapeutic relationship: A neglected association? Psychiatric Services, 62(5), 471-476. https://doi.org/10.1176/ps.62.5.pss6205_ 0471 
See it. Speak it. Write it. Change it.

Shin, S., Park, J. H., \& Bae, S. H. (2018). Nurse staffing and nurse outcomes: A systematic review and meta-analysis. Nursing Outlook, 66(3), 273-282. https://doi.org/10.1016/j.outlook.2017.1 2.002

Slemon, A., Jenkins, E., \& Bungay, V. (2017). Safety in psychiatric inpatient care: The impact of risk management culture on mental health nursing practice. Nursing Inquiry, 24(4), e12199. https://doi.org/10.1111/nin.12199

Soininen, P., Putkonen, H., Joffe, G., Korkeila, J., Puukka, P., Pitkänen, A., \& Välimäki, M. (2013). Does experienced seclusion or restraint affect psychiatric patients' subjective quality of life at discharge? International Journal of Mental Health Systems, 7. https://doi.org/10.1186/1752-4458-7-28

Stastny, P. (2000). Involuntary psychiatric interventions: A breach of the Hippocratic oath? Ethical Human Sciences and Services, 2(1), 21-41. https://pubmed.ncbi.nlm.nih.gov/155864 $52 /$

Stewart, D., Robson, D., Chaplin, R., Quirk, A., \& Bowers, L. (2012). Behavioural antecedents to pro re nata psychotropic medication administration on acute psychiatric wards. International Journal of Mental Health Nursing, 21(6), 540549. https://doi.org/10.1111/j.14470349.2012.00834.X

Streubert, H., \& Carperter, D. (2011). Designing data generation and management strategies. In Qualitative Research in Nursing:Advancing The Humanistic Imperative (pp. 33-55). Lippincott Co.

van Manen, M. (1997). From meaning to method. Qualitative Health Research, 7(3), 345-369.

Wilson, C., Rouse, L., Rae, S., \& Kar Ray, M. (2017). Is restraint a 'necessary evil' in mental health care? Mental health inpatients' and staff members' experience of physical restraint. International Journal of Mental Health Nursing, 26(5), 500-512. https://doi.org/10.1111/inm.12382
Zeller, S. L. (2017). The End of Chemical Restraints : Expert Opinion. Psychiatric Advisor, May, 32-34.

https://www.psychiatryadvisor.com/hom e/practice-management/the-end-ofchemical-restraints-expert-opinion/ 